\title{
Pacific
}

Journal of

Mathematics

\section{REFINED KATO INEQUALITIES FOR HARMONIC FIELDS ON KÄHLER MANIFOLDS}

Daniel Cibotaru and Peng Zhu 


\title{
REFINED KATO INEQUALITIES FOR HARMONIC FIELDS ON KÄHLER MANIFOLDS
}

\author{
Daniel Cibotaru And Peng Zhu
}

\section{We obtain a refined Kato inequality for closed and coclosed differential $(p, q)$-forms on a Kähler manifold.}

\section{Introduction}

Kato inequalities have been shown to be important technical tools which are used to prove analytic-geometric results. Branson [2000] and Calderbank, Gauduchon and Herzlich [2000] studied injectively elliptic Stein-Weiss operators and they showed that the sections in the kernel of such an operator satisfy improved Kato inequalities with constants that can be determined from representation theoretic data.

Calderbank et al. [2000, Theorem 6.3 (ii), case $k=1$ ] proved such an inequality for differential forms in the kernel of the Hodge-de Rham operator, $d+d^{*}$, on a Riemannian manifold. This result was also stated by Wang [2002] as Lemma 4.2 which, unfortunately, contains an omission, namely the condition that the degree has to be at most half the dimension of the manifold (which is what the author actually needs).

The purpose of this article is to further refine this Kato inequality for forms of type $(p, q)$ on a Kähler manifold. We obtain this refinement in Theorem 4.1 for all values of $p$ and $q$ except for $p=q$. When $p=q$ there is no improvement to the Riemannian case. The most important consequence of Theorem 4.1 is, in our view, a Kato inequality for holomorphic forms on all Kähler manifolds (see Corollary 4.3).

Our presentation follows closely the methods of Branson, Calderbank, Gauduchon and Herzlich. In fact, in the first part of this article, we will present a proof of the mentioned result from [Calderbank et al. 2000] avoiding as much as possible representation theoretic technicalities. It is this proof that our result in the Kähler case relies on.

The authors are supported by the CAPES (PNPD) program. The second author was partially supported by NSFC grants number 11101352 and 11071208 , and grant number 11026116 of the Tian Yuan Fund of Mathematics.

MSC2010: 53B05, 53B20, 53C55, 58J05.

Keywords: Kato inequality, Stein-Weiss operators, differential inequalities, harmonic forms. 
In the case of complete Kähler manifolds, Kong, Li and Zhou [2008] and Lam [2010] showed that an $L^{2}$ harmonic 1-form $\omega$ has to satisfy

$$
|d| \omega|| \leq \frac{1}{\sqrt{2}}|\nabla \omega| .
$$

In Corollary 4.9 we reprove this, making use of Theorem 4.1. Wang [2007] proved an inequality about real, closed and coclosed $(1,1)$-forms with a constant sharper than in the Riemannian case.

\section{Stein-Weiss operators}

Let $M$ be a Riemannian manifold of dimension $n$, not necessarily compact. We will call harmonic fields the forms $\omega \in C^{\infty}\left(M ; \Lambda^{k} T^{*} M\right)$ that satisfy $\left(d+d^{*}\right) \omega=0$. If $M$ is compact then the harmonic fields coincide with the harmonic forms, that is, solutions of $\Delta \omega=0$. This is still true if $M$ is complete in the presence of an $L^{2}$ integrability condition for $\omega$.

We introduce now the class of operators we will be interested in. Let $E$ be a vector space endowed with an inner product. We suppose that $E$ is a real representation of $\mathrm{SO}(n)$ given by $\rho: \mathrm{SO}(n) \rightarrow \mathrm{GL}(E)$ and let $F \hookrightarrow \mathbb{R}^{n} \otimes E$ be a subrepresentation of the canonical representation tensored with $E$. Let $\Pi$ be the orthogonal projection

$$
\Pi: \mathbb{R}^{n} \otimes E \rightarrow F .
$$

We will use the same letters to denote the projection of vector bundles over $M$,

$$
\Pi: T^{*} M \otimes E \rightarrow F
$$

The Levi-Civita connection on the frame bundle $P_{\mathrm{SO}} M$ of $M$ induces a connection $\nabla^{L C}$ on $E$.

Definition 2.1. A Stein-Weiss (gradient) operator is a first order differential operator $L: \Gamma(E) \rightarrow \Gamma(F)$ obtained as the composition

$$
\Gamma(E) \rightarrow \Gamma\left(T^{*} M \otimes E\right) \rightarrow \Gamma(F), \quad L:=\Pi \circ \nabla^{L C} .
$$

Remark 2.2. Branson [1997] made a beautiful study of Stein-Weiss operators and classified those operators that are injectively elliptic (see Definition 3.3).

Since a Stein-Weiss operator is essentially built from two objects, an orthogonal projection morphism of $\mathrm{SO}(n)$ representations and a connection on the manifold, by a slight abuse of terminology and notation we will talk about the composition of these two objects instead of the more lengthy expression "the composition of the connection with the associated projection of vector bundles". 
Definition 2.3. The rescaled Hodge-de Rham operator is the operator that acts on $k$-forms as

$$
\frac{1}{\sqrt{k+1}} d+\frac{1}{\sqrt{n+1-k}} d^{*} \text {. }
$$

Notice that the harmonic fields can be seen as the solutions of the rescaled Hodge-de Rham equation.

Proposition 2.4. The rescaled Hodge-de Rham operator is Stein-Weiss.

Proof. We will show that

$$
\Pi: \mathbb{R}^{n} \otimes \Lambda^{k} \mathbb{R}^{n} \rightarrow \Lambda^{k+1} \mathbb{R}^{n} \oplus \Lambda^{k-1} \mathbb{R}^{n}, \quad \eta \otimes \omega \mapsto\left(\frac{1}{\sqrt{k+1}} \eta \wedge \omega, \frac{-1}{\sqrt{n-k+1}} \iota_{\eta^{*}} \omega\right)
$$

is a morphism of $\mathrm{SO}(n)$ representations, where $\iota_{\eta^{*}}$ represents contraction with the metric dual to $\eta$. In fact, $\Pi$ is the orthogonal projection of the tensor product representation $\mathbb{R}^{n} \otimes \Lambda^{k} \mathbb{R}^{n}$ onto a direct sum of two subrepresentations.

Let $\left\{e_{i}, i=1 \ldots n\right\}$ be an orthogonal basis of $\mathbb{R}^{n}$. Let $\theta_{1}: \Lambda^{k+1} \mathbb{R}^{n} \rightarrow \mathbb{R}^{n} \otimes \Lambda^{k} \mathbb{R}^{n}$ be given by

$$
\theta_{1}\left(v_{1} \wedge \ldots \wedge v_{k+1}\right)=\frac{1}{\sqrt{k+1}} \sum_{i=1}^{k+1}(-1)^{i-1} v_{i} \otimes v_{1} \wedge \ldots \wedge \hat{v}_{i} \wedge \ldots \wedge v_{k+1}
$$

and $\theta_{2}: \Lambda^{k-1} \mathbb{R}^{n} \rightarrow \mathbb{R}^{n} \otimes \Lambda^{k} \mathbb{R}^{n}$ by

$$
\theta_{2}(\omega)=-\frac{1}{\sqrt{n-k+1}} \sum_{i=1}^{n} e_{i} \otimes\left(e_{i} \wedge \omega\right),
$$

be two linear maps. It is easy to check that they are morphisms of $\mathrm{SO}(n)$-representations. The first one is obviously so, while the second can be described as the composition of

$$
\mathbb{R}^{n} \otimes \mathbb{R}^{n} \otimes \Lambda^{k-1} \mathbb{R}^{n} \rightarrow \mathbb{R}^{n} \otimes \Lambda^{k} \mathbb{R}^{n}, \quad \xi \otimes \eta \otimes \omega \mapsto \xi \otimes \eta \wedge \omega
$$

with

(2-3) $\quad \Lambda^{k-1} \mathbb{R}^{n} \rightarrow \mathbb{R}^{n} \otimes \mathbb{R}^{n} \otimes \Lambda^{k-1} \mathbb{R}^{n}, \quad \omega \mapsto-\frac{1}{\sqrt{n-k+1}}\left(\sum_{i=1}^{n} e_{i} \otimes e_{i}\right) \otimes \omega$.

Note that if $A=\left(a_{i j}\right)_{1 \leq i, j, \leq n} \in \mathrm{SO}(n)$ then

$$
\sum_{i=1}^{n} A e_{i} \otimes A e_{i}=\sum_{i=1}^{n} \sum_{j, k=1}^{n} a_{j i} a_{k i} e_{j} \otimes e_{k}=\sum_{j, k=1}^{n}\left(A A^{T}\right)_{j k} e_{j} \otimes e_{k}=\sum_{i=1}^{n} e_{i} \otimes e_{i},
$$

which describes (2-3) (up to a constant) as the tensor product of the inclusion of the trivial representation into $\mathbb{R}^{n} \otimes \mathbb{R}^{n}\left(\right.$ via $\left.1 \mapsto \sum e_{i} \otimes e_{i}\right)$ with the identity on $\Lambda^{k-1} \mathbb{R}^{n}$. 
The following relations are straightforward

$$
\Pi_{1} \circ \theta_{1}=\mathrm{id}_{\Lambda^{k+1} \mathbb{R}^{n}} \quad \text { and } \quad \Pi_{2} \circ \theta_{2}=\mathrm{id}_{\Lambda^{k-1} \mathbb{R}^{n}},
$$

where $\Pi_{1}$ and $\Pi_{2}$ are the components of $\Pi$. Hence $\theta_{1}$ and $\theta_{2}$ are injective and $\Pi_{1}$ and $\Pi_{2}$ are surjective.

The presence of the constants $1 / \sqrt{k+1}$ and $1 / \sqrt{n-k+1}$ in the definition of $\theta_{1}$ and $\theta_{2}$, which a posteriori determines the constants for $\Pi$, is a reminder of the fact that we are looking for isometric monomorphisms of representations.

The next relations are essential:

$$
\operatorname{Ker} \Pi_{1}=\theta_{1}\left(\Lambda^{k+1} \mathbb{R}^{n}\right)^{\perp} \quad \text { and } \quad \operatorname{Ker} \Pi_{2}=\theta_{2}\left(\Lambda^{k-1} \mathbb{R}^{n}\right)^{\perp} .
$$

Indeed, using the identities from Lemma 2.5 (below) one can prove the $\subset$ inclusions which is enough because $\Pi_{1}$ and $\Pi_{2}$ are surjective.

Another easy application of Lemma 2.5 shows that the images of $\theta_{1}$ and $\theta_{2}$ are orthogonal and we deduce that $\Pi$ is the orthogonal projection onto the $\operatorname{SO}(n)$ invariant subspace $\Lambda^{k+1} \mathbb{R}^{n} \oplus \Lambda^{k-1} \mathbb{R}^{n-1} \hookrightarrow \mathbb{R}^{n} \otimes \Lambda^{k} \mathbb{R}^{n}$. Hence, $\Pi \circ \nabla^{L C}$ is a Stein-Weiss operator.

Berline et al. [1992] show in Proposition 1.22 that

$$
d=\sqrt{k+1} \Pi_{1} \circ \nabla^{L C},
$$

while their Proposition 2.8 shows that

$$
d^{*}=\sqrt{n-k+1} \Pi_{2} \circ \nabla^{L C} .
$$

This finishes the proof.

Lemma 2.5. (a) If $\xi, \eta_{i} \in \mathbb{R}^{n}$ and $\omega \in \Lambda^{k} \mathbb{R}^{n}$, then

$$
\left\langle\xi \wedge \omega, \eta_{1} \wedge \ldots \wedge \eta_{k+1}\right\rangle=\sum_{i=1}^{k+1}(-1)^{i-1}\left\langle\xi, \eta_{i}\right\rangle \cdot\left\langle\omega, \eta_{1} \wedge \ldots \hat{\eta}_{i} \ldots \wedge \eta_{k+1}\right\rangle .
$$

(b) If $\xi \in \mathbb{R}^{n}, \theta \in \Lambda^{k-1} \mathbb{R}^{n}$ and $\omega \in \Lambda^{k} \mathbb{R}^{n}$, then

$$
\left\langle\iota_{\xi}(\omega), \theta\right\rangle=\sum_{i=1}^{n}\left\langle\xi, e_{i}\right\rangle \cdot\left\langle\omega, e_{i} \wedge \theta\right\rangle .
$$

Proof. Let $I \subset\{1, \ldots, n\}$ be a subset with either $k+1$ or $k-1$ elements and let $e_{I}:=\bigwedge_{i \in I} e_{i}$. By linearity, it is enough to prove the identities for $\eta_{1} \wedge \ldots \wedge \eta_{k+1}=e_{I}$ and $\theta=e_{I}$, respectively. Also it is enough to consider $\omega=\alpha_{1} \wedge \ldots \wedge \alpha_{k}$.

In this situation, the number on the left hand side of the first identity is the $(k+1) \times(k+1)$ minor formed by taking the $I$-columns in the $n \times(k+1)$ matrix having the entries of $\xi$ on the first row and $\alpha_{1}, \ldots, \alpha_{k}$ on the next ones. The identity itself states the well known fact that this minor can be computed as an alternating 
sum of the relevant entries of $\xi$ multiplied with the corresponding $k \times k$ minors with entries from the matrix made of $\alpha_{1}, \ldots, \alpha_{k}$.

For the second identity, we further simplify by letting $\xi:=e_{p}$. Then we have to prove that

$$
\sum_{i=1}^{k}(-1)^{i-1}\left\langle e_{p}, \alpha_{i}\right\rangle \cdot\left\langle\alpha_{1} \wedge \ldots \hat{\alpha}_{i} \ldots \wedge \alpha_{k}, e_{I}\right\rangle=\left\langle\alpha_{1} \wedge \ldots \wedge \alpha_{k}, e_{p} \wedge e_{I}\right\rangle,
$$

which is nothing but the first identity for $\xi=e_{p}$ and $\omega=e_{I}$.

A natural question is whether the above rescaling of the Hodge-de Rham operator is the only one that turns it into a Stein-Weiss operator. This is true up to some signs.

Lemma 2.6. Let $\sigma: V \rightarrow W$ be a linear map between inner product spaces (real or complex). Let $\alpha: W \hookrightarrow V$ be an isometry such that $a \sigma \circ \alpha=\mathrm{id}_{W}$ for some constant $a$ and $\sigma(v)=0$ for all $v \in \alpha(W)^{\perp}$. Suppose there exists another constant $b$ and an isometry $\beta: W \hookrightarrow V$ such that $b \sigma \circ \beta=\mathrm{id}_{W}$ and $\sigma(v)=0$ for all $v \in \beta(W)^{\perp}$. Then $b=\mu a$ where $|\mu|=1$.

Proof. The conditions in the lemma express the fact that $a \sigma$ and $b \sigma$ are orthogonal projections onto $\alpha(W)$ and $\beta(W)$, respectively. Let $A, B: V \rightarrow V$ be the orthogonal projections seen as endomorphisms of $V$,

$$
A:=a \alpha \circ \sigma \quad \text { and } B:=b \beta \circ \sigma .
$$

Now, there exists an orthogonal transformation $T: V \rightarrow V$ such that $\beta=T \circ \alpha$; hence

$$
B=(b / a) T \circ A .
$$

The relation $B=B^{*}$ implies that $(b / a) A \circ T=(\bar{b} / \bar{a}) T^{*} \circ A$, which fed into

$$
B^{2}=\left(\frac{b}{a}\right)^{2} T \circ(A \circ T) \circ A=B
$$

gives

$$
\frac{|b|^{2}}{|a|^{2}} A^{2}=\frac{|b|^{2}}{|a|^{2}} A=B
$$

Hence the image of $B$ is the same as the image of $A$ and since they are orthogonal projections we must have $A=B$ and so $|b|^{2}=|a|^{2}$.

Corollary 2.7. The operators

$$
\pm \frac{1}{\sqrt{k+1}} d \quad \text { and } \quad \pm \frac{1}{\sqrt{n-k+1}} d^{*}
$$

are the only multiples of $d$ and $d^{*}$ which are Stein-Weiss. 


\section{Kato constants}

The classical Kato inequality states that if $\phi$ is a section of the vector bundle $E$ then the following inequality holds away from the set $\phi^{-1}(0)$ :

$$
|d| \phi|| \leq|\nabla \phi| .
$$

The equality above takes place when $\nabla \phi=\xi \otimes \phi$ for some 1-form $\xi$.

Definition 3.1. A refined Kato inequality is an inequality of the type

$$
|d| \phi|| \leq \alpha|\nabla \phi|
$$

with $\alpha<1$, which will be called a refined Kato constant.

The main insight of Branson [2000] and Calderbank et al. [2000] is that if $\phi$ is a section in the kernel of an injectively elliptic Stein-Weiss operator $L$ then $\phi$ satisfies a Kato inequality which is stronger than the classical one. Moreover the refined Kato constant $\alpha$ depends only on the symbol of $L$.

We give now the relevant definitions. We will work in a slightly more general context than in the previous section, that is, the bundle $E$ will be complex and $L$ will be a complex differential operator. The Stein-Weiss operators we considered above are real operators. However by complexifying the representations one can obtain complex operators. They should really be called $\mathrm{SO}(n)$ (real or complex) Stein-Weiss operators since they are associated to the $\mathrm{SO}(n)$-frame bundle on the manifold. In the next section we will consider $U(n)$ Stein-Weiss operators when the manifold $M$ is Kähler.

Definition 3.2. The symbol of a complex differential operator $L: \Gamma(E) \rightarrow \Gamma(F)$ of order $k$ is the map

$$
\sigma(L): T^{*} M \rightarrow \operatorname{Hom}(E, F), \quad \sigma(L)(\xi \otimes \ldots \otimes \xi):=i^{k} \frac{1}{k !}[\ldots[L, f], \ldots, f],
$$

where $\xi:=d f$. The symbol of a real differential operator is the symbol of its complexification.

The symbol of the Stein-Weiss operator $L: \Gamma(E) \rightarrow \Gamma(F)$ is the morphism of bundles

$$
i \Pi_{F}: T^{*} M \rightarrow \operatorname{Hom}(E, F) .
$$

The symbol of the formal adjoint $L^{*}: \Gamma(F) \rightarrow \Gamma(E)$ is

$$
\sigma\left(L^{*}\right): T^{*} M \rightarrow \operatorname{Hom}(F, E), \quad \sigma\left(L^{*}\right)=\sigma(L)^{*}=-i \Pi_{F}^{*}
$$

and the symbol of $L^{*} L$ is $\Pi_{F}^{*} \Pi_{F}$. 
Definition 3.3. An operator $L$ is called injectively elliptic if $L^{*} L$ is elliptic. Let $L$ be an operator of order 1. A number $\epsilon$ is called a constant of ellipticity for $L^{*} L$ if the following relation holds:

$$
\left\langle\sigma_{\xi}\left(L^{*} L\right)(v), v\right\rangle \geq \epsilon|\xi|^{2}|v|^{2}, \quad \text { for all } \xi \in T^{*} M, v \in E .
$$

If $L^{*} L$ is elliptic then the positivity of $\sigma\left(L^{*} L\right)$ implies that an ellipticity constant exists, at least locally. In the case of an injectively elliptic Stein-Weiss operator this constant is smaller than 1 because of the next straightforward lemma and the fact that the Bochner Laplacian $\nabla^{*} \nabla$ has constant of ellipticity equal to 1 .

Lemma 3.4. Let $F^{\perp}$ be the orthogonal complement of $F$ in $\mathbb{R}^{n} \otimes E$ and let $L_{\perp}$ be the corresponding Stein-Weiss operator. Then

$$
L^{*} L+L_{\perp}^{*} L_{\perp}=\nabla^{*} \nabla .
$$

Proof. One uses the equality $\nabla=L \oplus L_{\perp}$.

Here is the connection between constants of ellipticity and refined Kato constants:

Lemma 3.5. Let $\mathbb{C}^{n} \otimes E=F \oplus F^{\perp}$ and let $\Pi$ and $\Pi^{\perp}$ be the corresponding orthogonal projections onto $F$ and $F^{\perp}$ respectively. If $\phi \in \operatorname{Ker}(\Pi \circ \nabla)$ then

$$
|d| \phi|| \cdot|\phi| \leq|\nabla \phi| \cdot\left|\Pi^{\perp}\left(\xi_{0} \otimes \phi\right)\right|
$$

for some 1-form $\xi_{0}$ which is real and of norm 1.

Proof. We have

$$
d|\phi|^{2}=2 \operatorname{Re}\langle\nabla \phi, \phi\rangle .
$$

Let $\xi_{0}:=d|\phi|^{2} /\left.|d| \phi\right|^{2} \mid$. Then

$$
\begin{aligned}
2|d| \phi|| \cdot|\phi| & =\left.|d| \phi\right|^{2} \mid=\left\langle d|\phi|^{2}, \xi_{0}\right\rangle=2\left\langle\operatorname{Re}\langle\nabla \phi, \phi\rangle, \xi_{0}\right\rangle \\
& \stackrel{*}{=} 2 \operatorname{Re}\left\langle\langle\nabla \phi, \phi\rangle, \xi_{0}\right\rangle=2 \operatorname{Re}\left\langle\nabla \phi, \xi_{0} \otimes \phi\right\rangle=2 \operatorname{Re}\left\langle\Pi^{\perp} \circ \nabla(\phi), \xi_{0} \otimes \phi\right\rangle \\
& =2 \operatorname{Re}\left\langle\nabla \phi, \Pi^{\perp}\left(\xi_{0} \otimes \phi\right)\right\rangle \leq 2|\nabla \phi| \cdot\left|\Pi^{\perp}\left(\xi_{0} \otimes \phi\right)\right|,
\end{aligned}
$$

where the equality $*$ holds because $\xi_{0}$ is real.

Notice that

$$
\begin{aligned}
|\phi|^{2} & =\left|\xi_{0} \otimes \phi\right|^{2}=\left|\Pi\left(\xi_{0} \otimes \phi\right)\right|^{2}+\left|\Pi^{\perp}\left(\xi_{0} \otimes \phi\right)\right|^{2} \\
& =\left\langle\Pi^{*} \Pi\left(\xi_{0} \otimes \phi\right), \xi_{0} \otimes \phi\right\rangle+\left|\Pi^{\perp}\left(\xi_{0} \otimes \phi\right)\right|^{2},
\end{aligned}
$$

where $\Pi^{*} \Pi$ is the symbol of $L^{*} L$. So if $L^{*} L$ is injectively elliptic with a constant of ellipticity $\epsilon$ then

$$
\left|\Pi^{\perp}\left(\xi_{0} \otimes \phi\right)\right|^{2} \leq(1-\epsilon)|\phi|^{2} .
$$


In combination with Lemma 3.5 we have:

Proposition 3.6. Let $L$ be an injectively elliptic Stein-Weiss operator. If $\epsilon$ is a constant of ellipticity for $L^{*} L$, then $\alpha=\sqrt{1-\epsilon}$ is a refined Kato constant for $\phi \in \operatorname{Ker} L$.

Lemma 3.7. A constant of ellipticity for the rescaled Hodge-de Rham operator acting on $k$-forms is

$$
\epsilon=\left\{\begin{array}{cl}
1 & \text { if } k=0, n \\
\min \left\{\frac{1}{k+1}, \frac{1}{n-k+1}\right\} & \text { if } 1 \leq k \leq n-1 .
\end{array}\right.
$$

Proof. Let $1 \leq k \leq n-1$. If $L=\frac{1}{\sqrt{k+1}} d+\frac{1}{\sqrt{n+1-k}} d^{*}$ then

$$
L^{*} L=\frac{1}{k+1} d^{*} d+\frac{1}{n+1-k} d d^{*} \geq \min \left\{\frac{1}{k+1}, \frac{1}{n-k+1}\right\} \Delta
$$

and the Laplacian has constant of ellipticity 1 . For $k=0$ and $k=n$ the operator $L^{*} L$ is just the Laplacian on functions and on top degree forms, respectively.

A second simple proof can be provided using the symbol of $L^{*} L$ and the Cartan formula

$$
e_{u} \iota_{u}+\iota_{u} e_{u}=|u|^{2} \mathrm{id},
$$

where $e_{u}$ and $i_{u}$ are exterior multiplication and contraction by $u$, respectively.

Putting together Proposition 3.6 and Lemma 3.7 we have [Calderbank et al. 2000, Theorem 6.3 (ii), case $k=1$ ].

Theorem 3.8 (Calderbank-Gauduchon-Herzlich). Let $\omega$ be a $k$-form in the kernel of $d+d^{*}$. Then $\omega$ satisfies the refined Kato inequality

$$
\begin{aligned}
|d| \omega|| & \leq \sqrt{\frac{n-k}{n-k+1}}|\nabla \omega| & \text { if } 1 \leq k \leq n / 2, \\
|d| \omega|| & \leq \sqrt{\frac{k}{k+1}}|\nabla \omega| & \text { if } n / 2 \leq k \leq n-1,
\end{aligned}
$$

while for $k=0, n$ the form $\omega$ is parallel.

Remark 3.9. The question of sharpness in the previous inequalities depends in general on the manifold under consideration. For example, if the manifold $M$ is compact and symmetric then every harmonic field is parallel, hence the best constant in this case is 0 .

In general, to have equality above one first needs equality in Lemma 3.5 which after a quick inspection implies that the form $\omega$ has to satisfy the relation

$$
\nabla \omega=\xi \otimes \omega-\theta_{1}\left(\frac{1}{\sqrt{k+1}} \xi \wedge \omega\right)-\theta_{2}\left(-\frac{1}{\sqrt{n-k+1}} \iota \xi(\omega)\right)
$$


for some 1-form $\xi$, where $\theta_{1}$ and $\theta_{2}$ were defined in (2-1) and (2-2). More importantly, $\omega$ has to be a harmonic field. On the other hand, Branson [2000, Theorem 7] shows that such a form exists on flat $\mathbb{R}^{n}$. In his proof, it is essential that $E$ is an irreducible representation of $\mathrm{SO}(n)$. Branson's example is not $L^{2}$ integrable, hence it is conceivable that the inequalities above can be further refined if one imposes such a global condition.

Remark 3.10. Notice that the inequalities in the previous theorem respect Poincaré duality in the sense that the same refined Kato constant works both for $k$ and for $(n-k)$-forms. One has to expect this because of the next basic result.

Lemma 3.11. The star Hodge operator is an isometry and a parallel endomorphism of $\Lambda^{*} T^{*} M$.

Proof. The fact that it is an isometry is standard. To prove that it is parallel one first easily shows that the volume form dvol is parallel. This follows by differentiating

$$
|\mathrm{dvol}|^{2}=1 .
$$

which implies that $\left\langle\nabla_{X} \mathrm{dvol}, \mathrm{dvol}\right\rangle=0$ for all vector fields $X$. Then we apply $\nabla_{X}$ to the following pointwise equality which defines the Hodge star operator

$$
\eta \wedge * \omega=\langle\eta, \omega\rangle \text { dvol for all } \eta, \omega \in \Gamma\left(\Lambda^{k} T^{*} M\right)
$$

to get

$$
\nabla_{X} \eta \wedge * \omega+\eta \wedge \nabla_{X}(* \omega)=(X\langle\eta, \omega\rangle) \mathrm{dvol}+\langle\eta, \omega\rangle \nabla_{X} \mathrm{dvol}
$$

Hence $\eta \wedge\left(\nabla_{X} * \omega\right)=\left\langle\eta, \nabla_{X} \omega\right\rangle$ dvol $=\eta \wedge * \nabla_{X} \omega$, for all $\eta, \omega \in \Gamma\left(\Lambda^{k} T^{*} M\right)$.

Remark 3.12. The theory does not provide an inequality for harmonic forms, only for forms $\omega$ in the kernel of $d+d^{*}$, the so called harmonic fields. However in conjunction with an $L^{2}$ bound on $\omega$ one knows (see Proposition 4.6) that the harmonic fields are the same as the harmonic forms which is the case, for example, when $M$ is complete.

\section{The Kähler case}

On a Kähler manifold, using essentially the same theory, we get a better Kato constant for harmonic fields that respects Hodge duality. In what follows, $M$ is a Kähler manifold of complex dimension $n$. Notice that we can talk about the unitary frame bundle of $M$ and about $U(n)$ Stein-Weiss operators which are defined exactly as in Section 2 by replacing $\mathrm{SO}(n)$-representations with $U(n)$-representations and morphisms thereof.

Our result is as follows. 
Theorem 4.1. Let $0 \leq p, q \leq n$ and let $\omega \in \Gamma\left(\Lambda^{p, q} T^{*} M\right)$ such that $\left(d+d^{*}\right) \omega=0$. Let $\alpha \geq 0$ be such that $\alpha^{2}:=\frac{1}{2}$ if $p \in\{0, n\}$ or $q \in\{0, n\}$, and

$$
\alpha^{2}:=\min \left\{\max \left\{\frac{2 p+1}{2 p+2}, \frac{2 n-2 p+1}{2 n-2 p+2}\right\}, \max \left\{\frac{2 q+1}{2 q+2}, \frac{2 n-2 q+1}{2 n-2 q+2}\right\}\right\}
$$

otherwise. Then

$$
|d| \omega|| \leq \alpha|\nabla \omega|
$$

Proof. We write the decompositions

$$
d=\partial+\bar{\partial} \quad \text { and } \quad d^{*}=\partial^{*}+\bar{\partial}^{*} .
$$

Notice that since $\omega \in \Gamma\left(\Lambda^{p, q} T^{*} M\right)$, the condition $\left(d+d^{*}\right) \omega=0$ is equivalent to

$$
\partial \omega=\partial^{*} \omega=\bar{\partial} \omega=\bar{\partial}^{*} \omega=0 .
$$

We will see that

$$
L_{1}:=\frac{1}{\sqrt{p+1}} \partial+\frac{1}{\sqrt{n-p+1}} \partial^{*} \quad \text { and } \quad L_{2}:=\frac{1}{\sqrt{q+1}} \bar{\partial}+\frac{1}{\sqrt{n-q+1}} \bar{\partial}^{*}
$$

are $U(n)$ Stein-Weiss operators. Since the manifold is Kähler, each of the vector bundles $\Lambda^{p, q} T^{*} M$ comes endowed with a Levi-Civita connection. One can write down the symbols of each of the operators $\partial, \bar{\partial}, \partial^{*}, \bar{\partial}^{*}$ (compare with Proposition 3.67 in [Berline et al. 1992]):

$$
\begin{aligned}
\sigma(\partial): T^{*} M \rightarrow \operatorname{End}\left(\Lambda^{p, q} T^{*} M, \Lambda^{p+1, q} T^{*} M\right), & & \sigma(\partial)_{\xi}(\omega)=i \xi^{1,0} \wedge \omega, \\
\sigma(\bar{\partial}): T^{*} M \rightarrow \operatorname{End}\left(\Lambda^{p, q} T^{*} M, \Lambda^{p, q+1} T^{*} M\right), & & \sigma(\bar{\partial})_{\xi}(\omega)=i \xi^{0,1} \wedge \omega, \\
\sigma\left(\partial^{*}\right): T^{*} M \rightarrow \operatorname{End}\left(\Lambda^{p, q} T^{*} M, \Lambda^{p-1, q} T^{*} M\right), & & \sigma\left(\partial^{*}\right)_{\xi}(\omega)=(-i) \iota_{\left(\xi^{0,1}\right)^{*}}(\omega), \\
\sigma\left(\bar{\partial}^{*}\right): T^{*} M \rightarrow \operatorname{End}\left(\Lambda^{p, q} T^{*} M, \Lambda^{p, q-1} T^{*} M\right), & & \sigma\left(\bar{\partial}^{*}\right)_{\xi}(\omega)=(-i) \iota_{\left(\xi^{1,0}\right)^{*}}(\omega),
\end{aligned}
$$

where $\iota$ represents contraction and $\left(\left(\xi^{0,1}\right)^{*},\left(\xi^{1,0}\right)^{*}\right) \in T^{1,0} M \oplus T^{0,1} M$ is the metric dual of $\xi=\left(\xi^{1,0}, \xi^{0,1}\right) \in T_{\mathbb{C}}^{*} M$. Notice that for $\xi \in T^{*} M$ we have $\xi^{0,1}=\overline{\xi^{1,0}}$.

We want to show that $(-i) 1 / \sqrt{p+1} \sigma(\partial)$ when seen as a linear map defined on $T_{\mathbb{C}}^{*} M$ is an associated bundle morphism to an orthogonal projection of $U(n)$ representations. Analogous statements hold for the other three maps.

Let $\overline{\mathbb{C}}^{n}=\left(\mathbb{R}^{2 n},-i\right)$ be the conjugate of the standard complex space. The standard action of $U(n)$ is complex linear on $\overline{\mathbb{C}}^{n}$ and the standard Hermitian metric on $\mathbb{C}^{n}$ builds an isomorphism of $U(n)$ representations between $\overline{\mathbb{C}}^{n}$ and $\left(\mathbb{C}^{n}\right)^{*}$. The bundles $\Lambda^{p, q} T^{*} M:=\Lambda^{p} T^{1,0} M^{*} \otimes \Lambda^{q} T^{0,1} M^{*}$ are associated bundles to the unitary frame bundle of $M$ (induced by the Riemannian metric and the complex structure) and the canonical representations of $U(n)$ on $\Lambda^{p} \overline{\mathbb{C}}^{n} \otimes \Lambda^{q} \mathbb{C}^{n}$. 
Let $e_{i} \in \mathbb{C}^{n}, i=1, \ldots, n$ be an orthonormal basis with respect to the standard Hermitian metric and denote by $\bar{e}_{i} \in \overline{\mathbb{C}}^{n}, i=1, \ldots, n$ the dual, or conjugate, basis. We define

$$
\begin{aligned}
& \theta^{\partial}: \Lambda^{p+1} \overline{\mathbb{C}}^{n} \otimes \Lambda^{q} \mathbb{C}^{n} \hookrightarrow\left(\overline{\mathbb{C}}^{n} \oplus \mathbb{C}^{n}\right) \otimes \Lambda^{p} \overline{\mathbb{C}}^{n} \otimes \Lambda^{q} \mathbb{C}^{n}, \\
& \theta^{\bar{\partial}}: \Lambda^{p} \overline{\mathbb{C}}^{n} \otimes \Lambda^{q+1} \mathbb{C}^{n} \hookrightarrow\left(\overline{\mathbb{C}}^{n} \oplus \mathbb{C}^{n}\right) \otimes \Lambda^{p} \overline{\mathbb{C}}^{n} \otimes \Lambda^{q} \mathbb{C}^{n}, \\
& \theta^{\partial^{*}}: \Lambda^{p-1} \overline{\mathbb{C}}^{n} \otimes \Lambda^{q} \mathbb{C}^{n} \hookrightarrow\left(\overline{\mathbb{C}}^{n} \oplus \mathbb{C}^{n}\right) \otimes \Lambda^{p} \overline{\mathbb{C}}^{n} \otimes \Lambda^{q} \mathbb{C}^{n}, \\
& \theta^{\bar{\partial}^{*}}: \Lambda^{p} \overline{\mathbb{C}}^{n} \otimes \Lambda^{q-1} \mathbb{C}^{n} \hookrightarrow\left(\overline{\mathbb{C}}^{n} \oplus \mathbb{C}^{n}\right) \otimes \Lambda^{p} \overline{\mathbb{C}}^{n} \otimes \Lambda^{q} \mathbb{C}^{n},
\end{aligned}
$$

by

$\theta^{\partial}\left(\omega_{1} \wedge \ldots \wedge \omega_{p+1} \otimes \eta\right)=\frac{1}{\sqrt{p+1}} \sum_{i=1}^{p+1}(-1)^{i-1}\left(\omega_{i}, 0\right) \otimes \omega_{1} \wedge \ldots \hat{\omega}_{i} \ldots \wedge \omega_{p+1} \otimes \eta$, $\theta^{\bar{\partial}}\left(\omega \otimes \eta_{1} \wedge \ldots \wedge \eta_{q+1}\right)=\frac{1}{\sqrt{q+1}} \sum_{i=1}^{q+1}(-1)^{i-1}\left(0, \eta_{i}\right) \otimes \omega \otimes \eta_{1} \wedge \ldots \hat{\eta}_{i} \ldots \wedge \eta_{q+1}$,

$$
\begin{aligned}
& \theta^{\partial^{*}}(\omega \otimes \eta)=-\frac{1}{\sqrt{n-p+1}} \sum_{i=1}^{n}\left(0, e_{i}\right) \otimes \bar{e}_{i} \wedge \omega \otimes \eta, \\
& \theta^{\bar{\partial}^{*}}(\omega \otimes \eta)=-\frac{1}{\sqrt{n-q+1}} \sum_{i=1}^{n}\left(\bar{e}_{i}, 0\right) \otimes \omega \otimes e_{i} \wedge \eta .
\end{aligned}
$$

These intertwiners, just as in the Riemannian case, are isometric monomorphisms of $U(n)$ representations. One easily checks the relations

$$
\begin{array}{ll}
\frac{-i}{\sqrt{p+1}} \sigma(\partial) \circ \theta^{\partial}=\mathrm{id}_{\Lambda^{p+1} \overline{\mathbb{C}}^{n} \otimes \Lambda^{q} \mathbb{C}^{n}}, & \frac{-i}{\sqrt{n-p+1}} \sigma\left(\partial^{*}\right) \circ \theta^{\partial^{*}}=\mathrm{id}_{\Lambda^{p-1} \overline{\mathbb{C}}^{n} \otimes \Lambda^{q} \mathbb{C}^{n}}, \\
\frac{-i}{\sqrt{q+1}} \sigma(\bar{\partial}) \circ \theta^{\bar{\partial}}=\mathrm{id}_{\Lambda^{p} \overline{\mathbb{C}}^{n} \otimes \Lambda^{q+1} \mathbb{C}^{n}}, & \frac{-i}{\sqrt{n-q+1}} \sigma\left(\bar{\partial}^{*}\right) \circ \theta^{\bar{\partial}^{*}}=\mathrm{id}_{\Lambda^{p} \overline{\mathbb{C}}^{n} \otimes \Lambda^{q-1} \mathbb{C}^{n} .} .
\end{array}
$$

Here the symbol maps $\sigma(\partial), \sigma\left(\partial^{*}\right), \sigma(\bar{\partial}), \sigma\left(\bar{\partial}^{*}\right)$ are the obvious maps between vector spaces corresponding to the morphisms of vector bundles above.

Using Lemma 2.5 (which works in the Hermitian case as well) one can check that

$$
\begin{array}{ll}
\operatorname{Ker} \sigma(\partial)=\operatorname{Im}\left(\theta^{\partial}\right)^{\perp}, & \operatorname{Ker} \sigma\left(\partial^{*}\right)=\operatorname{Im}\left(\theta^{\partial^{*}}\right)^{\perp} \\
\operatorname{Ker} \sigma(\bar{\partial})=\operatorname{Im}\left(\theta^{\bar{\partial}}\right)^{\perp}, & \operatorname{Ker} \sigma\left(\bar{\partial}^{*}\right)=\operatorname{Im}\left(\theta^{\bar{\partial}^{*}}\right)^{\perp} .
\end{array}
$$

Combining this with the fact that the images of $\theta^{\partial}$ and $\theta^{\partial^{*}}$ are orthogonal and an analogous statement about $\theta^{\bar{\partial}}$ and $\theta^{\bar{\partial}^{*}}$, one gets the claim about $L_{1}$ and $L_{2}$. 
The constants of ellipticity for $L_{1}^{*} L_{1}$ and $L_{2}^{*} L_{2}$ are easy to compute from the relations

$$
\partial^{*} \partial+\partial \partial^{*}=\bar{\partial}^{*} \bar{\partial}+\bar{\partial} \bar{\partial}^{*}=\frac{1}{2} \Delta .
$$

Hence for $p \notin\{0, n\}$ the constant for $L_{1}^{*} L_{1}$ is $\frac{1}{2} \min \{1 /(p+1), 1 /(n-p+1)\}$ and similarly for $q \notin\{0, n\}$ the constant for $L_{2}^{*} L_{2}$ is $\frac{1}{2} \min \{1 /(q+1), 1 /(n-q+1)\}$.

When $p \in\{0, n\}$ then $L_{1}^{*} L_{1}=\frac{1}{2} \Delta$ and the constant is $\frac{1}{2}$. Similarly for $q \in\{0, n\}$, $L_{2}^{*} L_{2}=\frac{1}{2} \Delta$. One now chooses the smaller Kato constant from the ones provided by the inequalities induced by $L_{1}$ and by $L_{2}$.

Remark 4.2. The Kato constants in the Kähler case provided by Theorem 4.1 for a harmonic field $\omega$ of bidegree $(p, q)$ are smaller or equal than the Kato constants provided by Theorem 3.8 except in the case $p=q$.

A special case of the theorem is:

Corollary 4.3. If $\omega \in \Gamma\left(\Lambda^{p, 0} M\right)$ is a holomorphic p-form on a Kähler manifold then it satisfies the Kato inequality

$$
|d| \omega|| \leq \frac{1}{\sqrt{2}}|\nabla \omega| .
$$

Proof. The operator $L_{2}=\bar{\partial}$ is injectively elliptic Stein-Weiss on $\Gamma\left(\Lambda^{p, 0} M\right)$ and the constant of ellipticity for $L_{2}^{*} L_{2}$ is $1 / 2$.

The following result characterizes the equality case in Theorem 4.1 in the simplest of the situations.

Proposition 4.4. Let $\omega \in \Gamma\left(\Lambda^{0, q}\right)$ and $\eta \in \Gamma\left(\Lambda^{p, 0}\right)$ be harmonic fields on a Kähler manifold of dimension $n$. A necessary condition for equality for $\omega$ and $\eta$ in Theorem 4.1 is the existence of real one-forms $\xi, \gamma \in \Gamma\left(T^{*} M\right)$ such that

$$
\nabla \omega=\xi^{0,1} \otimes \omega \text { and } \nabla \eta=\gamma^{1,0} \otimes \eta .
$$

If $\omega \in \Gamma\left(\Lambda^{n, q}\right)$ and $\eta \in \Gamma\left(\Lambda^{p, n}\right)$ then a necessary condition for equality is

$$
\nabla \omega=\xi^{1,0} \otimes \omega \text { and } \nabla \eta=\gamma^{0,1} \otimes \eta .
$$

Proof. The equality in Lemma 3.5 happens when there exists a real valued function $f: M \rightarrow \mathbb{R}$ such that

$$
\nabla \omega=f \Pi^{\perp}\left(\xi_{0} \otimes \omega\right)=f \xi_{0} \otimes \omega-\Pi\left(f \xi_{0} \otimes \omega\right) .
$$

Let $\xi:=f \xi_{0}$. This is a real 1-form. In the case $\omega \in \Gamma\left(\Lambda^{0, q}\right)$, the operator $\Pi$ is nothing else but $\xi \otimes \omega \mapsto \xi^{1,0} \wedge \omega$ where the element $\xi^{1,0} \wedge \omega$ is a section of $T_{\mathbb{C}}^{*} M \otimes \Lambda^{0, q}$ via the map

$$
\theta^{\partial}\left(\xi^{1,0} \wedge \omega\right)=\xi^{1,0} \otimes \omega
$$


We have therefore the first claim and the second is entirely analogous. The third claim follows from the observation that

$$
\sum_{i=1}^{n}\left(0, e_{i}\right) \otimes \bar{e}_{i} \wedge \iota_{\left(\xi^{0,1}\right)^{*}} \omega=\xi^{0,1} \otimes \omega,
$$

and the last claim is similar to the third one.

One might ask whether this is the best we can do in the Kähler case with this technique. The following lemma is a possible answer.

Lemma 4.5. If a linear combination $a \partial+b \partial^{*}+c \bar{\partial}+d \bar{\partial}^{*}$ is a $U(n)$ Stein-Weiss operator acting on $(p, q)$ forms then either $c=d=0$ or $a=b=0$ or $b=d=0$ or $a=c=0$. If the operator is also injectively elliptic then, up to some constants of absolute value 1, the operators $L_{1}$ and $L_{2}$ in Theorem 4.1 are the only possibilities. Proof. The first thing to note is that the images of $\theta^{\partial^{*}}$ and $\theta^{\bar{\partial}}$ are not orthogonal. On the other hand, by the complex version of Lemma 2.6, the morphisms of representations $\theta^{\partial^{*}}$ and $\theta^{\bar{\partial}}$ are determined up to a constant of modulus 1 by the symbols of the operators $\partial^{*}$ and $\bar{\partial}$. Hence if a linear combination of the operators $a \partial+b \partial^{*}+c \bar{\partial}+d \bar{\partial}^{*}$ is Stein-Weiss then either $b$ or $c$ has to be 0 . The same dichotomy goes for $\partial$ and $\bar{\partial}^{*}$ and hence either $a$ or $d$ has to be zero.

One can check that an operator $a \partial+c \bar{\partial}$ is not injectively elliptic and the same for $b \partial^{*}+d \bar{\partial}^{*}$ for any values of $a, b, c$ and $d$. Another use of Lemma 2.6 shows that $L_{1}$ and $L_{2}$ (up to some constants of absolute value 1, which do not influence the constants of ellipticity) are the only injectively elliptic $U(n)$ Stein Weiss linear combinations of $\partial, \partial^{*}, \bar{\partial}$ and $\bar{\partial}^{*}$.

Notice that the inequalities in Theorem 4.1 hold without any global condition on $\omega$ or $M$. We would like now to reprove the result about 1 -forms mentioned in the introduction which appears in [Kong et al. 2008; Lam 2010] using Theorem 4.1. We have almost everything except for the fact that a harmonic 1-field splits into harmonic $(1,0)$ and $(0,1)$ components. The following proposition, which is well known in the case of compact Kähler manifolds takes care of that (compare with Proposition 1 in [Yau 1976]).

Proposition 4.6. Let $M$ be a complete Kähler manifold and let $\omega \in \Gamma\left(\Lambda^{k} T^{*} M\right)$ be $L^{2}$ integrable. The following equations are equivalent:

(1) $\left(d+d^{*}\right) \omega=0$.

(2) $\left(\partial+\partial^{*}\right) \omega=0$.

(3) $\left(\bar{\partial}+\bar{\partial}^{*}\right) \omega=0$.

Proof. The following identities hold on any Kähler manifold:

$$
\Delta=2 \Delta_{\partial}=2 \Delta_{\bar{\partial}},
$$


where $\Delta=\left(d+d^{*}\right)^{2}, \Delta_{\partial}=\left(\partial+\partial^{*}\right)^{2}$ and $\Delta_{\bar{\partial}}=\left(\bar{\partial}+\bar{\partial}^{*}\right)^{2}$.

If $M$ is compact then it is standard that the solutions of the Laplace equation $\Delta \omega=0$ satisfy $\left(d+d^{*}\right) \omega=0$ and similarly for the other two. If $M$ is complete, we will show that $\Delta_{\partial} \omega=0$ and $\omega \in L^{2}$ imply that $\left(\partial+\partial^{*}\right) \omega$ is $L^{2}$ integrable.

This is enough to allow us to plug in $\eta=\left(\partial+\partial^{*}\right) \omega$ in the following relation

$$
\int_{M}\left\langle\left(\partial+\partial^{*}\right) \eta, \omega\right\rangle=\int_{M}\left\langle\eta,\left(\partial+\partial^{*}\right) \omega\right\rangle
$$

(and deduce that, in fact, $\left.\left(\partial+\partial^{*}\right) \omega=0\right)$ because (4-2) holds whenever the forms $\eta, \omega,\left(\partial+\partial^{*}\right) \eta,\left(\partial+\partial^{*}\right) \omega$ are all in $L^{2}$. This is due to the fact that $\partial+\partial^{*}$ is formally self-adjoint.

We will be using a special collection of cut-off functions $\psi_{\nu}$ with the following properties: there exists an exhaustion of compact subsets $K_{v} \subset K_{v+1}^{\circ} \subset M$ such that

$$
\psi_{\nu} \equiv 1 \text { on } K_{v}, \quad\left|d \psi_{v}\right| \leq 1 \text { on } M \quad \text { and } \quad \operatorname{supp} \psi_{v} \subset K_{v+1} .
$$

Such a collection exists on a complete manifold by the Hopf-Rinow theorem, see Proposition 8.1 in [Demailly 1996]. We have

$$
\begin{aligned}
0 & =\int_{M}\left\langle\psi_{\nu}^{2} \omega, \Delta_{\partial} \omega\right\rangle=\int_{M}\left\langle\partial\left(\psi_{\nu}^{2} \omega\right), \partial \omega\right\rangle+\int_{M} \psi_{\nu}^{2}\left|\partial^{*} \omega\right|^{2} \\
& =2 \int_{M}\left\langle\partial \psi_{\nu} \wedge \omega, \psi_{\nu} \partial \omega\right\rangle+\int_{M} \psi_{\nu}^{2}|\partial \omega|^{2}+\int_{M} \psi_{\nu}^{2}\left|\partial^{*} \omega\right|^{2} .
\end{aligned}
$$

It follows from here that

$$
\int_{M} \psi_{\nu}^{2}|\partial \omega|^{2} \leq 2\left|\int_{M}\left\langle\partial \psi_{\nu} \wedge \omega, \psi_{\nu} \partial \omega\right\rangle\right| \leq 2\left(\int_{M}\left|\partial \psi_{\nu} \wedge \omega\right|^{2}\right)^{1 / 2}\left(\int_{M}\left|\psi_{\nu}\right|^{2}|\partial \omega|^{2}\right)^{1 / 2}
$$

We get

$$
\int_{M} \psi_{v}^{2}|\partial \omega|^{2} \leq 4 \int_{M}\left|\partial \psi_{v} \wedge \omega\right|^{2} \leq C \int_{M}\left|\partial \psi_{v}\right|^{2}|\omega|^{2} \leq C \int_{M}|\omega|^{2},
$$

where in the last inequality we used (4-3). Therefore, by the monotone convergence theorem, $\partial \omega$ is $L^{2}$ integrable and by (4-4), $\partial^{*} \omega$ is also $L^{2}$ integrable.

Remark 4.7. After the article was accepted for publication we became aware of Wolf's theorem [Lawson and Michelsohn 1989, Chapter II, Theorem 5.7], which says that on any complete Riemannian manifold, any Dirac operator $D$ has a unique self-adjoint extension and $\operatorname{Ker} D=\operatorname{Ker} D^{2}$ in the space $L^{2}$ sections on which $D$ acts. The equality of kernels follows essentially the same steps as the proof of Proposition 4.6 and we decided to keep this proof for completeness.

We need the following property: 
Lemma 4.8. Let $\phi_{1} \in \Gamma\left(E_{1}\right)$ and $\phi_{2} \in \Gamma\left(E_{2}\right)$ be sections of two vector bundles, $E_{1}$ and $E_{2}$, that satisfy the Kato inequalities

$$
|d| \phi_{1}|| \leq \alpha_{1}\left|\nabla^{E_{1}} \phi_{1}\right| \quad \text { and } \quad|d| \phi_{2}|| \leq \alpha_{2}\left|\nabla^{E_{2}} \phi_{2}\right|,
$$

for some constants $\alpha_{1}$ and $\alpha_{2} \leq 1$. Then $\left(\phi_{1}, \phi_{2}\right) \in \Gamma\left(E_{1} \oplus E_{2}\right)$ satisfies the inequality

$$
|d|\left(\phi_{1}, \phi_{2}\right)|| \leq \max \left\{\alpha_{1}, \alpha_{2}\right\}\left|\nabla^{E_{1} \oplus E_{2}}\left(\phi_{1}, \phi_{2}\right)\right| .
$$

Proof. We calculate

$$
\begin{aligned}
2\left|\left(\phi_{1}, \phi_{2}\right)\right| \cdot|d|\left(\phi_{1}, \phi_{2}\right)|| & =\left.|d|\left(\phi_{1}, \phi_{2}\right)\right|^{2}|=| d\left|\phi_{1}\right|^{2}+d\left|\phi_{2}\right|^{2} \mid \\
& \leq 2\left|\phi_{1}\right| \cdot|d| \phi_{1}||+2\left|\phi_{2}\right| \cdot|d| \phi_{2}|| \\
& \leq 2 \max \left\{\alpha_{1}, \alpha_{2}\right\}\left(\left|\phi_{1}\right| \cdot\left|\nabla^{E_{1}} \phi_{1}\right|+\left|\phi_{2}\right| \cdot\left|\nabla^{E_{2}} \phi_{2}\right|\right) \\
& \leq 2 \max \left\{\alpha_{1}, \alpha_{2}\right\}\left|\left(\phi_{1}, \phi_{2}\right)\right| \cdot\left|\nabla^{E_{1} \oplus E_{2}}\left(\phi_{1}, \phi_{2}\right)\right| .
\end{aligned}
$$

Combining Theorem 4.1, Proposition 4.6 and Lemma 4.8 we get the following result that appears in [Kong et al. 2008; Lam 2010].

Corollary 4.9. Let $\omega$ be a harmonic form of degree 1 or $2 n-1$ that is $L^{2}$ integrable on a complete Kähler manifold $M$ of complex dimension $n$. Then

$$
|d| \omega|| \leq \frac{1}{\sqrt{2}}|\nabla \omega| .
$$

\section{Acknowledgements}

We thank Professor Detang Zhou for suggesting the problem to us and for useful conversations. We also thank X. Wang, P. Gauduchon and M. Herzlich for clarifications regarding their work and for useful suggestions. Por fim, agradecemos ao Professor Saponga Firmo pelo seu apoio.

\section{References}

[Berline et al. 1992] N. Berline, E. Getzler, and M. Vergne, Heat kernels and Dirac operators, Grundlehren der Math. Wiss. 298, Springer, Berlin, 1992. MR 94e:58130 Zbl 0744.58001

[Branson 1997] T. Branson, "Stein-Weiss operators and ellipticity", J. Funct. Anal. 151:2 (1997), 334-383. MR 99b:58219 Zbl 0904.58054

[Branson 2000] T. Branson, "Kato constants in Riemannian geometry", Math. Res. Lett. 7 (2000), 245-261. MR 2001i:58066 Zbl 1039.53033

[Calderbank et al. 2000] D. M. J. Calderbank, P. Gauduchon, and M. Herzlich, "Refined Kato inequalities and conformal weights in Riemannian geometry", J. Funct. Anal. 173:1 (2000), 214-255. MR 2001f:58046 Zbl 0960.58010

[Demailly 1996] J.-P. Demailly, " $L^{2}$ estimates for the $\bar{\partial}$-operator on complex manifolds", Lecture notes, Institut Fourier, 1996, Available at http://www-fourier.ujf-grenoble.fr/ demailly/books.html. 
[Kong et al. 2008] S. Kong, P. Li, and D. Zhou, "Spectrum of the Laplacian on quaternionic Kähler manifolds", J. Diff. Geom. 78:2 (2008), 295-332. MR 2008k:58065 Zbl 1140.58014

[Lam 2010] K.-H. Lam, "Results on a weighted Poincaré inequality of complete manifolds", Trans. Amer. Math. Soc. 362:10 (2010), 5043-5062. MR 2011f:53062 Zbl 1201.53041

[Lawson and Michelsohn 1989] H. B. Lawson, Jr. and M.-L. Michelsohn, Spin geometry, Princeton Mathematical Series 38, Princeton University Press, 1989. MR 91g:53001 Zbl 0688.57001

[Wang 2002] X. Wang, "On the $L^{2}$-cohomology of a convex cocompact hyperbolic manifold", Duke Math. J. 115:2 (2002), 311-327. MR 2003m:58048 Zbl 1221.58023

[Wang 2007] X. Wang, "On $L^{2}$ cohomology of ACH Kähler manifolds”, Proc. Amer. Math. Soc. 135:9 (2007), 2949-2960. MR 2008d:32020 Zbl 1123.53037

[Yau 1976] S. T. Yau, "Some function-theoretic properties of complete Riemannian manifold and their applications to geometry", Indiana Univ. Math. J. 25:7 (1976), 659-670. MR 54 \#5502 Zbl 0335.53041

Received May 6, 2011. Revised October 14, 2011.

DANIEl CiBotaru

Instituto dE MATEMÁtica e Estatística

UNIVERSIDADE FEDERAL FLUMINENSE

Rua Mário Santos Braga

24020-140 NITERÓI, RJ

BRAZIL

cibdan@yahoo.com

PENG ZHU

SCHOol of Mathematical SCIENCE

YANGZHOU UNIVERSITY

SIWANGTING ROAD 180

YANGZHOU, 225002

CHINA

zhupeng2004@yahoo.com.cn 


\title{
PACIFIC JOURNAL OF MATHEMATICS
}

\author{
http://pacificmath.org \\ Founded in 1951 by \\ E. F. Beckenbach (1906-1982) and F. Wolf (1904-1989)
}

\section{EDITORS}

V. S. Varadarajan (Managing Editor)

Department of Mathematics

University of California

Los Angeles, CA 90095-1555

pacific@math.ucla.edu

Vyjayanthi Chari

Department of Mathematics

University of California

Riverside, CA 92521-0135

chari@math.ucr.edu

\section{Robert Finn}

Department of Mathematics Stanford University

Stanford, CA 94305-2125

finn@math.stanford.edu

Kefeng Liu

Department of Mathematics

University of California

Los Angeles, CA 90095-1555

liu@math.ucla.edu
Darren Long

Department of Mathematics

University of California

Santa Barbara, CA 93106-3080

long@math.ucsb.edu

Jiang-Hua Lu

Department of Mathematics

The University of Hong Kong

Pokfulam Rd., Hong Kong jhlu@maths.hku.hk

Alexander Merkurjev

Department of Mathematics

University of California

Los Angeles, CA 90095-1555

merkurev@math.ucla.edu
Sorin Popa

Department of Mathematics University of California

Los Angeles, CA 90095-1555 popa@math.ucla.edu

Jie Qing

Department of Mathematics

University of California

Santa Cruz, CA 95064

qing@cats.ucsc.edu

Jonathan Rogawski

Department of Mathematics

University of California

Los Angeles, CA 90095-1555

jonr@math.ucla.edu

\section{PRODUCTION}

pacific@math.berkeley.edu

\section{SUPPORTING INSTITUTIONS}

ACADEMIA SINICA, TAIPEI

CALIFORNIA INST. OF TECHNOLOGY INST. DE MATEMÁTICA PURA E APLICADA KEIO UNIVERSITY

MATH. SCIENCES RESEARCH INSTITUTE NEW MEXICO STATE UNIV.

OREGON STATE UNIV.

\author{
STANFORD UNIVERSITY \\ UNIV. OF BRITISH COLUMBIA \\ UNIV. OF CALIFORNIA, BERKELEY \\ UNIV. OF CALIFORNIA, DAVIS \\ UNIV. OF CALIFORNIA, LOS ANGELES \\ UNIV. OF CALIFORNIA, RIVERSIDE \\ UNIV. OF CALIFORNIA, SAN DIEGO \\ UNIV. OF CALIF., SANTA BARBARA
}

\author{
UNIV. OF CALIF., SANTA CRUZ \\ UNIV. OF MONTANA \\ UNIV. OF OREGON \\ UNIV. OF SOUTHERN CALIFORNIA \\ UNIV. OF UTAH \\ UNIV. OF WASHINGTON \\ WASHINGTON STATE UNIVERSITY
}

These supporting institutions contribute to the cost of publication of this Journal, but they are not owners or publishers and have no responsibility for its contents or policies.

See inside back cover or pacificmath.org for submission instructions.

The subscription price for 2012 is US \$420/year for the electronic version, and \$485/year for print and electronic.

Subscriptions, requests for back issues from the last three years and changes of subscribers address should be sent to Pacific Journal of Mathematics, P.O. Box 4163, Berkeley, CA 94704-0163, U.S.A. Prior back issues are obtainable from Periodicals Service Company, 11 Main Street, Germantown, NY 12526-5635. The Pacific Journal of Mathematics is indexed by Mathematical Reviews, Zentralblatt MATH, PASCAL CNRS Index, Referativnyi Zhurnal, Current Mathematical Publications and the Science Citation Index.

The Pacific Journal of Mathematics (ISSN 0030-8730) at the University of California, c/o Department of Mathematics, 969 Evans Hall, Berkeley, CA 94720-3840, is published monthly except July and August. Periodical rate postage paid at Berkeley, CA 94704, and additional mailing offices. POSTMASTER: send address changes to Pacific Journal of Mathematics, P.O. Box 4163, Berkeley, CA 94704-0163.

PJM peer review and production are managed by EditFLOW ${ }^{\mathrm{TM}}$ from Mathematical Sciences Publishers.

PUBLISHED BY PACIFIC JOURNAL OF MATHEMATICS

at the University of California, Berkeley 94720-3840

A NON-PROFIT CORPORATION

Typeset in LATEX

Copyright $(02012$ by Pacific Journal of Mathematics 


\section{PACIFIC JOURNAL OF MATHEMATICS}

Volume $256 \quad$ No. $1 \quad$ March 2012

On slim double Lie groupoids

NiCOlas ANDruskiewitsch, JEsus OCHOA ARANGO and AlEJANDRO

TIRABOSCHI

Topological classification of quasitoric manifolds with second Betti number 2

19

Suyoung Choi, SeOnjeOng PARK and Dong Youp SuH

Refined Kato inequalities for harmonic fields on Kähler manifolds

DANiEL Cibotaru and PENG ZHU

Deformation retracts to the fat diagonal and applications to the existence of peak solutions of nonlinear elliptic equations

E. Norman Dancer, Jonathan Hillman and Angela Pistoia

Descent for differential Galois theory of difference equations: confluence and $q$-dependence

LuCia Di Vizio and Charlotte HaRdouin

Modulation and natural valued quiver of an algebra

FANG LI

Willmore hypersurfaces with two distinct principal curvatures in $\mathbb{R}^{n+1}$

TONGZHU LI

Variational inequality for conditional pressure on a Borel subset

YuAN Li, ERCAI CHEN and WEN-ChIAO CHENG

New homotopy 4-spheres

DANIEL NASH

Combinatorial constructions of three-dimensional small covers

YASUZO NISHIMURA

On a theorem of Paul Yang on negatively pinched bisectional curvature

AERYEONG SEO

Orders of elements in finite quotients of Kleinian groups

PETER B. Shalen

A new algorithm for finding an 1.c.r. set in certain two-sided cells

JIAN-YI SHI

Addendum to the article Superconnections and parallel transport

FLORIN DUMITRESCU 